\title{
Forced Response Analysis of a Fan with Boundary Layer Inlet Distortion
}

\author{
Milind A. Bakhle ${ }^{1}$ \\ NASA Glenn Research Center, Cleveland, Ohio 44135, USA \\ T. S. R. Reddy ${ }^{2}$ \\ University of Toledo, Toledo, Ohio 43606, USA \\ Rula M. Coroneos ${ }^{3}$ \\ NASA Glenn Research Center, Cleveland, Ohio 44135, USA
}

\begin{abstract}
Boundary layer ingesting propulsion systems have the potential to significantly reduce fuel burn for future generations of commercial aircraft, but these systems must be designed to overcome the challenge of high dynamic stresses in fan blades due to forced response. High dynamic stresses can lead to high cycle fatigue failures. High-fidelity computational analysis of the fan aeromechanics is integral to an ongoing effort to design a boundary layer ingesting inlet and fan for a wind-tunnel test. An unsteady flow solution from a Reynoldsaveraged Navier Stokes analysis of a coupled inlet-fan system is used to calculate blade unsteady loading and assess forced response of the fan to distorted inflow. Conducted prior to the mechanical design of a fan, the initial forced response analyses performed in this study provide an early look at the levels of dynamic stresses that are likely to be encountered. For the boundary layer ingesting inlet, the distortion contains strong engine order excitations that act simultaneously. The combined effect of these harmonics was considered in the calculation of the forced response stresses. Together, static and dynamic stresses can provide the information necessary to evaluate whether the blades are likely to fail due to high cycle fatigue. Based on the analyses done, the overspeed condition is likely to result in the smallest stress margin in terms of the mean and alternating stresses. Additional work is ongoing to expand the analyses to off-design conditions, on-resonance conditions, and to include more detailed modeling of the blade structure.
\end{abstract}

\section{Introduction}

$\mathrm{B}$ oundary layer ingestion (BLI) propulsion systems have been studied for the significant reduction in aircraft fuel burn that they offer ${ }^{1-6}$. System studies have shown that $5-10 \%$ reduction in fuel burn is possible. A detailed systems study ${ }^{7}$ focused on the propulsion system and its integration into a hybrid wing body aircraft has shown that a BLI aircraft mission fuel burn benefit up to $5 \%$ can be achieved for future aircraft relative to a baseline highperformance, pylon-mounted, propulsion system. Key technologies such as a low-loss inlet and a high-performance distortion-tolerant fan are necessary to achieve this significant fuel burn benefit. A BLI inlet ${ }^{8}$ has been designed as part of a research task to test a BLI propulsion system in a wind tunnel to simulate cruise conditions of a representative large commercial transport hybrid wing body aircraft. As part of the optimization of the inlet design, total pressure losses were reduced and distortion harmonics were also reduced. The reduction in distortion leads to a fan with minimal loss in performance relative to operation in clean undistorted flow. Moreover, a reduced distortion also alleviates the unsteady loading on the fan blades, which reduces the possibility of fatigue failure.

For a fan operating in a distorted flow, each blade traverses a circumferentially-varying flow resulting in a timevarying loading on the blade. This loading is somewhat similar to the loading that is present when an engine operates in an off-axis flow such as in crosswind or sideslip. The main differences are the large thickness of the boundary layer flow in a BLI propulsion system, the persistence of unsteady loading across all operating conditions,

\footnotetext{
${ }^{1}$ Aerospace Engineer, Multiscale and Multiphysics Modeling Branch, AIAA Associate Fellow.

${ }^{2}$ Senior Research Associate, Multiscale and Multiphysics Modeling Branch, AIAA Associate Fellow.

${ }^{3}$ Research Aerospace Engineer, Multiscale and Multiphysics Modeling Branch, AIAA Senior Member.
}

1

American Institute of Aeronautics and Astronautics 
and the specific harmonic content related to a BLI distortion. The unsteady loading acting on the fan blades leads to forced response vibrations and vibratory stresses. This paper describes in detail the initial forced response analyses performed on a boundary layer ingesting fan. The objectives of these analyses are to determine the static and dynamic stresses in the fan blade due to operation in a distorted inflow and to examine the concerns regarding high cycle fatigue failure due to a combination of mean and alternating stresses. The present aeromechanics analyses are performed at cruise flight conditions and further studies are required at other challenging conditions including takeoff.

\section{Fan Blade Structural Dynamics Analysis}

For a forced response analysis of the fan blade, it is necessary to develop a model for the structural dynamics and for the unsteady aerodynamics. In this section, the structural model and the structural dynamics analysis are described. An intermediate aerodynamic design iteration of the BLI fan is used to create a structural model for the fan blade. It is assumed that the fan blades are made of Titanium alloy Ti-6Al-4V, which is typical for scale model fan blades. Also, it is assumed that dovetail attachments will be used to hold the fan blades in the disk. Since a blade attachment design is not available, the blade root section is fully constrained in this analysis. In the structural model, 8node hexahedral or brick elements are used. Centrifugal and mean pressure loads are applied on the blade. Nonlinear static and modal analyses are performed using commercial finite element analysis software (MSC Nastran) to calculate the blade static deflection, static stress, modal deflections (mode shapes), modal stresses, and natural frequencies. The analyses include the effects of centrifugal stiffening and softening.

For the design speed, Fig. 1 shows the mean stress and deflection in the blade resulting from the rotational and mean pressure loads. The mode shape and frequency results for the first ten modes are shown in Fig. 2.

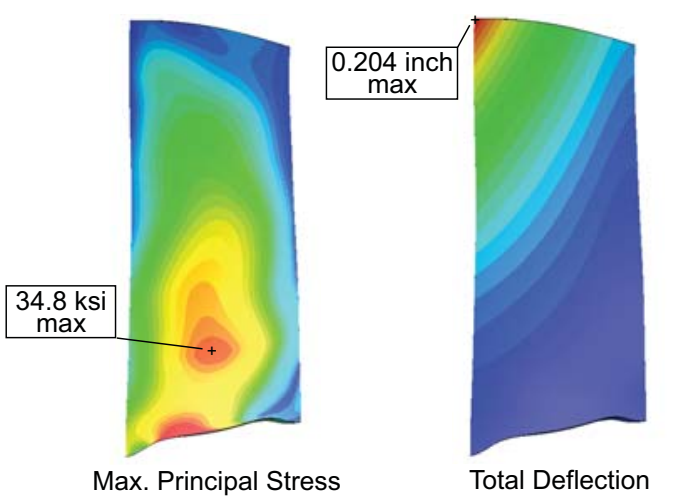

Figure 1. Fan blade mean stress and deflection due to rotation and mean pressure loading.
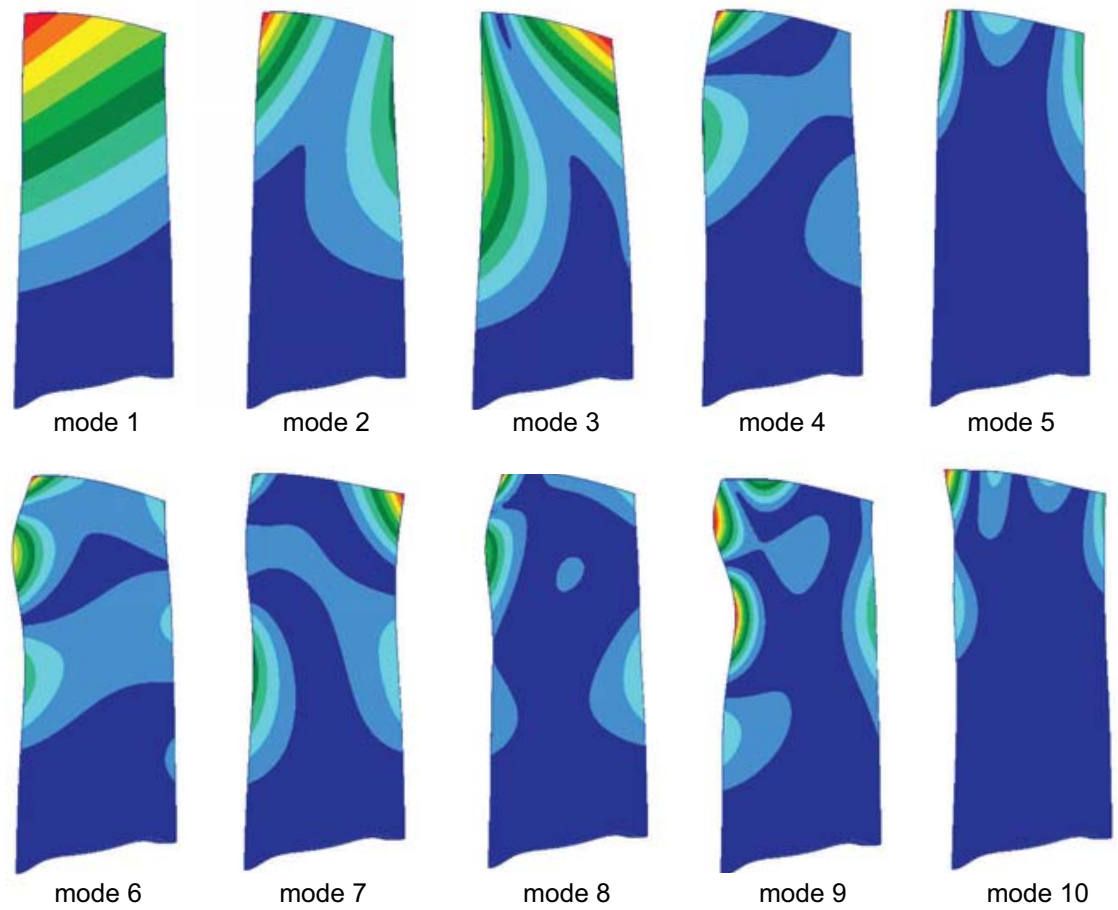

Figure 2. Fan blade vibration mode shapes at design speed. 


\section{Fan Aerodynamic Analysis}

In the present work, the fan aerodynamic analysis is based on a time-domain solution of Reynolds-averaged Navier Stokes (RANS) equations in a coupled inlet-fan domain. Details of the aerodynamic analysis are not provided in this paper. In the analysis performed, the fan blades do not vibrate or deform. The fan operates in a distorted flowfield resulting from the non-axisymmetric flow through the inlet duct upstream of the fan. As part of the unsteady flow solution, the unsteady pressure distribution on each fan blade is calculated and stored as a time history. The inlet distortion provides a periodic excitation to each blade with a fundamental period of one rotor revolution. Figure 3 shows the distribution of the unsteady pressure on the blade surface as the blade goes through one revolution. The mean, minimum, and maximum pressure are shown at four spanwise locations.

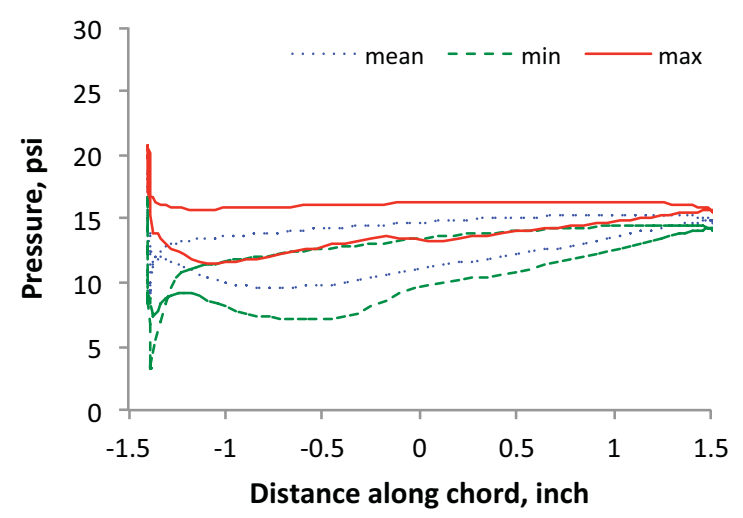

(a) $24 \%$ span

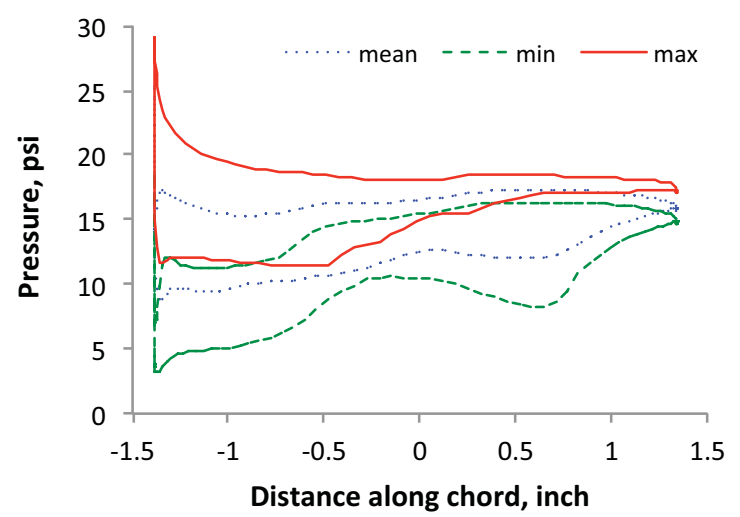

(c) $89 \%$ span

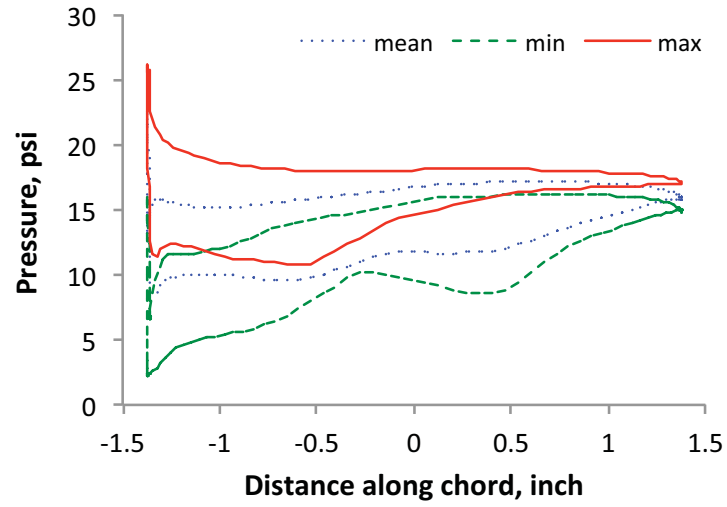

(b) $75 \%$ span

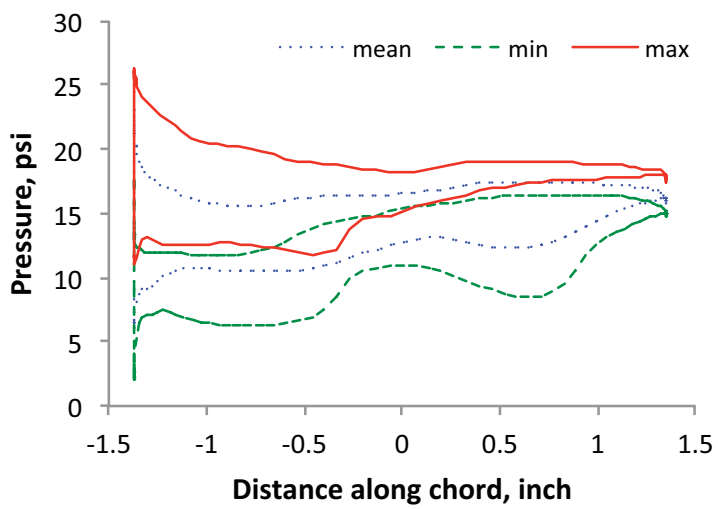

(d) $99 \%$ span

Figure 3. Blade pressure distribution with inlet distortion.

With the flowfield converged to periodicity, the unsteady pressure is resolved into harmonic components with the fundamental frequency equal to the rotational frequency of the fan. Based on the results of this decomposition, the first and second harmonics of pressure are significant in magnitude, with a rapid drop-off beyond the fourth harmonic. As shown in Fig. 4, the first four harmonics of integrated pressure on the blade have a significant magnitude. Other harmonics will also be important if the excitation frequency is close to a natural frequency as will be seen in the following section. 


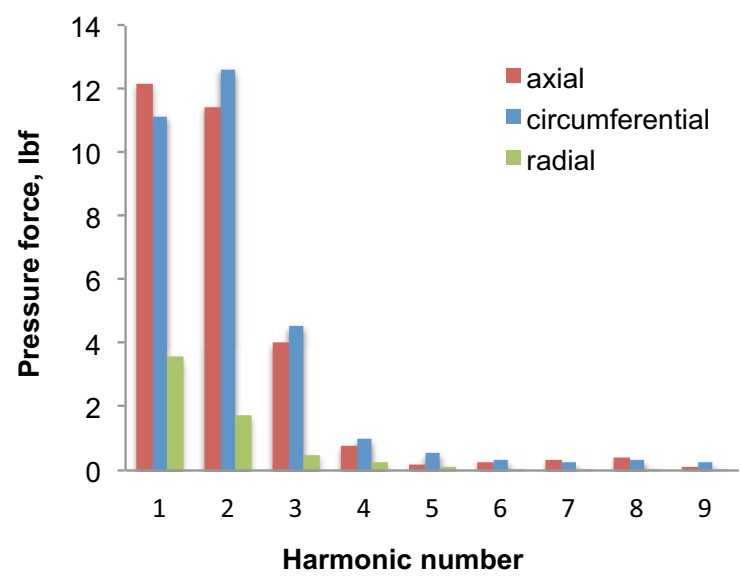

Figure 4. Harmonic components of integrated blade pressure.

\section{Fan Blade Forced Vibration Response}

The governing equations of blade structural dynamics are used to calculate the static and dynamic stresses at each engine order excitation. The unsteady aerodynamic pressure harmonic (both magnitude and phase) on the blade surface is prescribed as the loading. This forced response calculation is repeated for each engine order or harmonic. Note that damping is not included in this analysis and therefore the results are expected to be conservative. Before performing the forced response analysis, it can be noted that a large response is expected when the excitation frequency is nearly equal to a natural frequency. To determine such conditions, a Campbell diagram is constructed by repeating the modal analysis at different rotational speeds. Typical design practices would prescribe margins on frequency and/or rotational speed to avoid crossings on the Campbell diagram near the design operating condition. In the present case, mechanical design has not been carried out prior to this forced response analysis. Thus, the results presented here represent an early look at the forced response for a BLI fan subjected to distorted inflow.

Figure 5 shows the Campbell diagram for the fan rotor blade. The rotational speed is non-dimensionalized by the design value. The lines of constant slope are the engine order (E) excitation lines that correspond to the harmonic content of the excitation. For example, at the design rotational speed the third harmonic of unsteady loading acts as a $3 \mathrm{E}$ excitation with a frequency close to the mode 2 frequency. Similarly, the 7E excitation frequency is nearly equal to the mode 4 frequency. The margins between the blade natural frequency and the nearest excitation frequency are as follows: $37 \%$ margin for mode $1-1 \mathrm{E}$ crossing, $8 \%$ margin for mode $2-3 \mathrm{E}$ crossing, $11 \%$ margin for mode $3-4 \mathrm{E}$ crossing, $1.5 \%$ margin for mode $4-7 \mathrm{E}$ crossing. Note that mechanical design has not yet been carried out and typical design practices would require some of these margins to be increased. For example, a $10 \%$ margin on frequency may be required on crossings near the design rotational speed for a conventional fan design.

For various frequencies of excitation (fundamental frequency and harmonics), the unsteady aerodynamic loading is prescribed in the form of a complex-valued pressure distribution on the blade surface and the forced vibration response is calculated using a direct frequency response method available in commercial finite element structural analysis software. The vibration response results are obtained

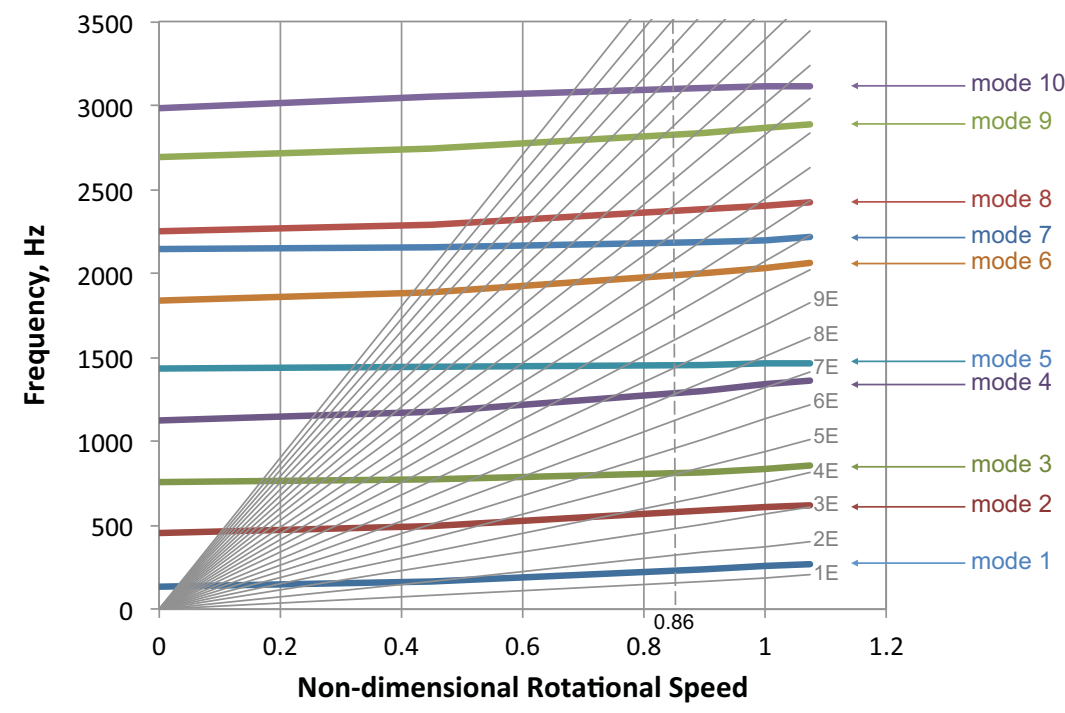

Figure 5. Campbell diagram for BLI fan blade.

4

American Institute of Aeronautics and Astronautics 
in the form of a displacement vector and a stress tensor. The forced response to an excitation at a single excitation frequency obtained with this approach includes contributions from various modes since the loading is applied directly on the structural model of the blade. An alternate approach is to rewrite the governing structural dynamics equations in a modal form and then calculate the response as a summation of responses in each mode as described in Ref. 9.

The results of primary interest are distributions of mean and alternating stresses in the blade that determine if the combined stress state will lead to fatigue failure at any location in the blade. The mean stress and deflection in the blade resulting from rotational and mean pressure loads at design speed were previously presented in Fig. 1. The distributions of alternating stresses, von Mises stresses as indicators of overall stress, due to the various engine order excitations are shown in Fig. 6. As noted earlier, the excitation level drops off rapidly after the third harmonic and accordingly the response drops off as well in general. However, small excitations with a frequency close to the natural frequency (near crossing on the Campbell diagram) can potentially result in a significant response. This can be observed in Fig. 6 for the 7E excitation that has a larger response compared to some other excitations due to the $7 \mathrm{E}$ frequency being close to the mode 4 natural frequency in Fig. 5 (1.5\% margin).

Since damping is not included in this initial analysis, it is important to clarify the potential impact of damping on the forced response and alternating stresses. In general, damping arises due to material hysteresis and friction at the blade root attachment. In addition, unsteady aerodynamic forces acting on the blade that are phase-shifted from the blade vibratory motion also can be characterized as aerodynamic damping. From a single-degree-of-freedom system analysis, it can be easily shown that the effect of damping is significant only near a Campbell crossing where the excitation frequency is close to the natural frequency. For the $1 \mathrm{E}$ excitation, the ratio of excitation frequency to natural frequency is 0.73 and thus a damping ratio of 0.02 ( $2 \%$ of critical damping) will only reduce the response and stress by $0.2 \%$. In contrast, for the $7 \mathrm{E}$ excitation, the ratio of excitation frequency to the mode 4 natural frequency is 0.985 and a damping ratio of 0.02 (2\% critical damping) can reduce the response by approximately $40 \%$. But, since the response is quite small due to the relatively low excitation amplitude, damping still does not affect the overall stress by much for this case.

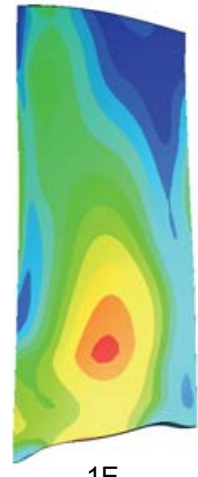

$1 \mathrm{E}$
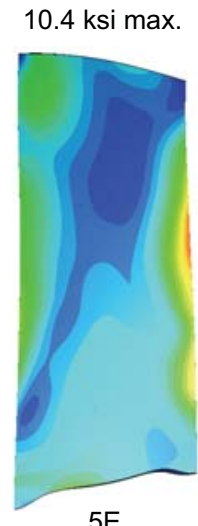

0.9 ksi max.

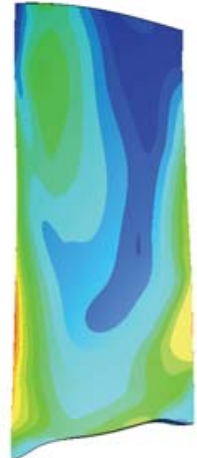

$2 \mathrm{E}$
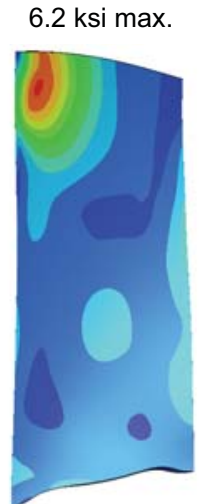

$6 \mathrm{E}$

$1.1 \mathrm{ksi} \max$.

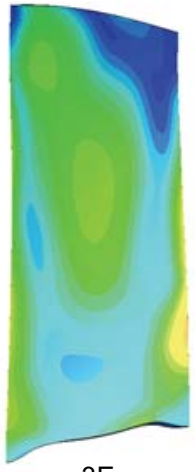

3E
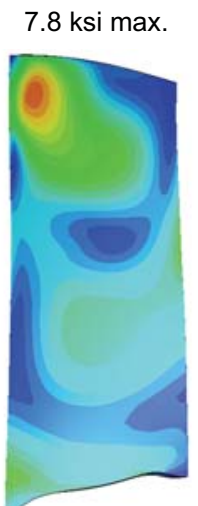

7E

3.7 ksi max.

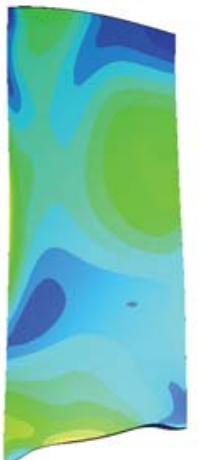

$4 \mathrm{E}$

$1.3 \mathrm{ksi}$ max.

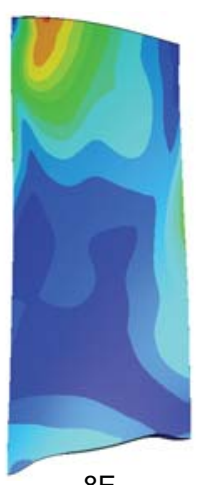

$8 \mathrm{E}$

$1.3 \mathrm{ksi} \max$.

Figure 6. Fan blade alternating stresses corresponding to various engine order excitations. 
